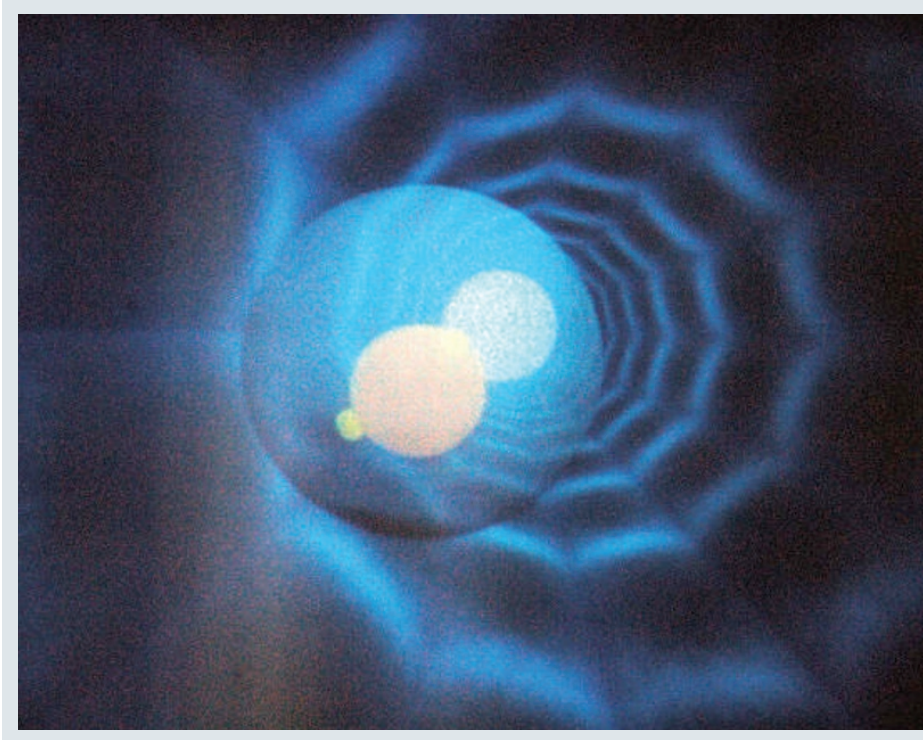

\title{
EXHIBITION
}

\section{Discovering the invisible}

What happens inside an accelerator when very highenergy particles collide? Visitors to the ALEXploratorium, the science centre within the Bibliotheca Alexandrina in Egypt, can now take a look for themselves (see picture).

An interactive exhibition, 'The Microscopes of Physics', introduces students and the broader public to both the smallest particles at the heart of atoms and the boundaries of the Universe. Successive rooms introduce visitors to the world of atoms and their constituent particles, as revealed by particle accelerators and detectors, and on to the cosmos and its origins in the Big Bang.

The exhibition has been designed and brought about by the Italian National Institute for Nuclear Physics as part of the Mediterranean Association for Science Communication, in which science communicators from southern Europe and North Africa join forces to spread scientific culture. The exhibition runs until 31 October 2006.

\section{Small science, big challenge}

\author{
Nanotechnology: New Promises, New \\ Dangers \\ by Toby Shelley \\ Zed Books: 2006. 208 pp. £45
}

\section{Julia A. Moore}

Toby Shelley's book Nanotechnology provides a short, accessible primer on the world of nanotechnology - the revolutionary realm of seeing, measuring, controlling and making things on the scale of atoms and molecules. It raises key questions about how this disruptive technology will affect human health, the environment, civil liberties, weaponry, and people in developing countries. Most important, it asks who decides which nanotechnology research projects will go forward, and who will profit from or be disadvantaged by the applications that result. It is big business: Lux Research reports that sales of products incorporating nanotechnology were worth US $\$ 32$ billion in 2005, and the US National Science Foundation predicts that nanotech-related goods and services could be a \$1-trillion market by 2015 .

This book is long on high-minded questions. The author, a journalist at the Financial Times, supports some worthy and necessary steps. For example, he says that when governments evaluate the risks and benefits of nanotechnology, they should consider the health and environment impacts of nanotech products over their full life cycle, including development, manufacture, transport and disposal. He also says they should develop appropriate and internationally applied regulation to mitigate the risks without stifling innovation, and weigh carefully any military uses and encroachments on personal freedom.

On the downside, there is not much here that's new. All the analysis and prescriptions have been given before, sometimes with more passion and insight. Much of the territory was covered by Bill Joy, the co-founder of Sun Microsystems, in his article 'Why the future doesn't need us' in Wired magazine in April 2000. And Nanotechnology: A Gentle Introduction to the Next Big Idea by Mark and Daniel Ratner (Prentice Hall, 2002) is a superior read. Shelley's book also contains some annoying minor errors and sweeping statements, particularly about other new technologies such as agricultural biotechnology, that many scientists will dispute.

In my view, nanotechnology will transcend today's Wall Street hype. It will eventually affect how people work, what they eat, how they communicate, and how long they live. It will change their medical care, energy sources, water and environment. As a result, its considerable benefits and possible risks need to be seriously and rigorously debated.

The progress made by science and technology continues to accelerate. Yet society seems increasingly incapable of answering questions, such as those raised in the 1970 s by Alvin Toffler, about how we should respond to it. In 1995, the US Congress unwisely dismantled the Office of Technology Assessment (OTA), one of the world's few organizations to provide policy-makers with authoritative analyses of the complex scientific and technical challenges of the late twentieth century and beyond.

In the week when I finished reading Shelley's book, I attended the Enrico Fermi Award ceremony at the US National Academy of Sciences. The winner of this $\$ 375,000$ prize was Arthur Rosenfeld, who developed technological solutions and applied innovative policies aimed at reducing energy consumption.

A news feature published last year in Nature (438, 410-412; 2005) described what is increasingly known in energy circles as the
'Rosenfeld effect'. This refers to the ingenious policies and programmes devised by Rosenfeld that have helped California - the world's sixth-largest economy - keep per capita electricity consumption steady since the early 1970 s, even though the state's economy was outpacing that of the United States as a whole. Today, California's per capita electricity use is the country's lowest, just over half that for the nation overall. Originally appointed by a Democrat, Rosenfeld was reappointed as a member of the California Energy Commission by Arnold Schwarzenegger, a Republican, when he became the state's governor.

Nanotechnologists need to find a way of cloning people such as Rosenfeld who are willing to roll up the sleeves of their lab coats and make good on science and engineering's promise to apply knowledge for the betterment of all mankind. The twenty-first century needs leaders like Benjamin Franklin who combine political and social vision with knowledge of science and engineering, and who understand how to move governments, public opinion, researchers and corporations to achieve true progress.

If nanotechnology is to be developed successfully, fresh kinds of policy insights, leadership and partnerships will be needed, alongside bold measures to create national and international assessment institutions that build on the OTA model. New global oversight mechanisms are required that can handle the complex convergence of nanotechnology, biotechnology, and information and cognitive science, and that are up to the task of 'taming the genie', as Shelley puts it in the final chapter.

Those looking for a roadmap to navigate this brave new world will find partial directions in Shelley's book. Hopefully, future books will help complete the journey.

Julia A. Moore is at the Woodrow Wilson

International Center for Scholars, 1300

Pennsylvania Avenue, Washington DC

20004-3027, USA. 